\title{
Use of cervical mucus to screen for gynecological malignancies: a pilot study
}

\author{
Ihab Lamzabi ${ }^{1}$, Lela Buckingham ${ }^{1}$, Mezgebe Gebrekiristos ${ }^{1}$, Richa Jain ${ }^{1}$, Paolo Gattuso ${ }^{1}$, \\ Vijaya Reddy ${ }^{1}$, Alfred Guirguis ${ }^{2}$, Summer Dewdney ${ }^{2}$, Jacob Rotmensch ${ }^{2}$ and Pincas Bitterman ${ }^{1}$ \\ ${ }^{1}$ Rush University Medical Center, Department of Pathology, Chicago, IL, USA and ${ }^{2}$ Rush University Medical \\ Center, Department of Obstetrics and Gynecology, Chicago, IL, USA
}

\begin{abstract}
High-grade malignancies are the leading cause of death from gynecological tumors. Unfortunately, no efficient screening method is available for these tumors. In this paper we report the results of a pilot study based on the frequency of TP53 mutations in these cancers. Mucus from the cervix of 32 hysterectomy specimens with no grossly visible cervical or serosal involvement were included in this study. TP53 exons 5-9 mutations were screened for mutations using single strand conformation polymorphism (SSCP). Immunostain for p53 protein was performed in all fallopian tubes and in a sample from the tumors that were identified prospectively. A total of 32 cases including 19 malignant, and 13 benign cases were included. P53 immunostain was positive in only 5 cases including 3 high grade malignant tumors and 2 precancerous lesions (serous tubal intraepithelial lesion or p53 signature) in the fallopian tubes. A TP53 mutation band pattern was detected by SSCP in 2/3 and 2/2 cases respectively. Twenty-seven cases were negative for p53 imunostain, 4 of which were positive for TP53 mutation by SSCP including 3 low-grade malignancies. The results of this study provide evidence that DNA from precursor lesions of high grade ovarian, fallopian tube and endometrial carcinomas can be detected in cervical mucus. Further studies using different markers, in preoperative setting and large scale screening studies will determine the utility of using cervical mucus to screen for gynecological malignancies. Modern Pathology (2013) 26, 1508-1513; doi:10.1038/modpathol.2013.92; published online 14 June 2013
\end{abstract}

Keywords: DNA; fallopian tube; gynecologic; mucus; ovary; screening

High-grade malignancies are the leading cause of death from gynecological tumors. ${ }^{1-4}$ These tumors can arise from different locations including peritoneum, ovaries, fallopian tubes and endometrium, and show molecular aberrations with TP53 gene mutation being the most common and one of the earliest events in their carcinogenesis. ${ }^{5-8}$

High-grade epithelial ovarian cancers are highly aggressive tumors that represent $75 \%$ of all ovarian carcinomas, and account for $90 \%$ of deaths. They display high chromosomal instability and show TP53 mutations in $>95 \%$ of cases. Recent studies suggest that most high-grade ovarian epithelial carcinomas arise from epithelial implantS originating from the fimbriated end of the fallopian tubes. In addition, high-grade epithelial ovarian tumors are commonly associated with tubal intraepithelial carcinomas and tubal p53 signature. Tubal intra-

Correspondence: Dr I Lamzabi, MD, Pathology Department, Rush University Medical Center, 1750 West Harrison Street, Room 570 Jelke, Chicago, IL 60612, USA.

E-mail: Ihab_Lamzabi@rush.edu

Received 19 January 2013; revised 1 April 2013; accepted 11 April 2013; published online 14 June 2013 epithelial carcinomas are positive for p53 immunostain, show epithelial stratification, high proliferative activity by Ki67 immunostain, and harbor TP53 mutation in most of the cases. P53 signature also called serous tubal intraepithelial lesion (STILs) shows positivity with p53 immunostain but does not show epithelial stratification or high proliferative activity. They are also associated with TP53 mutations in about $50 \%$ of the cases. ${ }^{6-10}$ Highgrade endometrial carcinomas also harbor TP53 mutations and have the worst prognosis among endometrial carcinomas. ${ }^{5}$

All high-grade gynecological malignancies share the tendency to become symptomatic only at advanced stages, especially the ovarian high-grade carcinomas. ${ }^{1-4}$ Interest in early detection has been centered on ovarian carcinomas in an effort to reduce their mortality and several large-scale studies have been undertaken. Most of these studies used serum tumor markers associated with ovarian malignancies (particularly CA125) and/or ultrasound as a screening method. However, Serum markers and ultrasound are not sensitive nor specific for carcinomas. Furthermore, some studies found that screening using these methods did not 
reduce the mortality from ovarian cancers. ${ }^{11,12}$ Similarly, screening for endometrial carcinoma using Pap smears or liquid based cytology showed low sensitivity. ${ }^{13}$

In this paper, we describe the results of a pilot study that may open a new era for the screening and early diagnosis of high-grade gynecological malignancies and perhaps all gynecological cancers. The idea behind this study is based on the usual path of an ovocyte from the ovary entering the uterus through the fallopian tubes, and perhaps ending most of the time in the cervical mucus. This path suggests that DNA molecules from neoplastic cells may also take the same route and be found in the cervical mucus. Testing the mucus has several advantages. First, getting a sample from the mucus is not invasive and has no side effects. Second, most women in the reproductive age are screened for cervical cancer, and the mucus can be taken at the same time as a Pap smear. Third, the viscosity of the mucus might help trap molecules from the upper gynecologic tract. Finally, the molecules in the mucus would provide information mainly about the gynecologic tract, which will make further investigation and surveillance more targeted.

When we began the study, there was no publication in the English literature about using cervical mucus as a screening tool for gynecological malignancies.

\section{Materials and methods}

\section{Case Selection}

With approval of the Rush Institutional Review Board, hysterectomy specimens received fresh for frozen section with suspected gynecological malignancies and a group of presumed benign cases received for routine surgical pathology were randomly selected from April 2012 to July 2012. The uteri from which samples were taken had to be intact with no grossly visible cervical or serosal tumor involvement to minimize direct contamination of the mucus.

\section{Histologic Examination}

Histologic sections stained with hematoxylin and eosin were examined by board certified pathologists. The pathologists were blinded and did not know which cases were included in the study.

\section{Immunohistochemistry}

Immunostain for p53 protein was performed using p53 monoclonal antibody (DAKO (M7001) clone DO-7) run at 1:1000 dilution with Leica's pretreatment of ER2 for 20 min, on Leica Bond III instrument. All fallopian tube sections and a sample from the tumors or precancerous lesions that were prospectively identified on histologic examination were stained. Only strong $(3+)$ positivity that was continuous and diffuse was considered positive by the authors (PB and IL).

\section{Mucus Sample Collection}

Mucus was collected by aspiration using a 10 cc syringe and then labeled with the patients identification and pathology number, snap frozen, and stored at $-86^{\circ} \mathrm{C}$.

\section{DNA Extraction}

DNA was extracted from 100-200 $\mu$ l of mucus using the 5 PRIME cell/tissue extraction buffers (5 PRIME, Inc., Gaithersburg, MD). The mucus was rinsed from the needle-less syringe tip with $600 \mu \mathrm{l}$ lysis buffer in a $35 \times 10 \mathrm{~mm}$ Petri Dish. The mixture was transferred to a $2 \mathrm{ml}$ microcentrifuge tube, using a transfer pipet, and incubated at $55^{\circ} \mathrm{C}$ for $4 \mathrm{~h}$ or until the solution cleared. Samples were placed on ice and cooled before adding $200 \mu \mathrm{l}$ protein precipitation buffer. After vigorous vortexing for $20 \mathrm{~s}$, the mixture was centrifuged at $12000 \mathrm{~g}$ for $5 \mathrm{~min}$. The supernatant was added to $500 \mu \mathrm{l}$ isopropanol in a $1.5 \mathrm{ml}$ microcentrifuge tube and mixed by inversion at least 50 times. The precipitated DNA was pelleted by centrifugation at $15000 \mathrm{~g}$ for $10 \mathrm{~min}$. The resulting pelleted DNA was rinsed one time by adding $500 \mu \mathrm{l} 70 \%$ ethanol and centrifuging at $15000 \mathrm{~g}$ for $5 \mathrm{~min}$. The tubes were inverted on absorbent paper for $10 \mathrm{~min}$ and then resuspended in 100-250 $\mu \mathrm{l}$ rehydration buffer, depending on the size of the pellet.

\section{TP53 Exons 5-9 Amplification}

TP53 exons 5-9 were amplified for each specimen using HotStarTaq (Qiagen, Valencia, CA). Each $25 \mu \mathrm{l}$ reaction mix contained $1 \mathrm{X}$ HotStarTaq buffer brought to $2 \mathrm{mM} \mathrm{MgCl}_{2}, 0.2 \mathrm{mM}$ dNTP mix, $0.1 \mu \mathrm{M}$ each forward and reverse primers (Table 1) and 1 unit HotStarTaq enzyme. Five $\mu$ l of template was added to each reaction mix and amplified in an ABI 9700 thermal cycler (Applied Biosystems, Foster City, CA) with the following program: $95^{\circ} \mathrm{C} 15 \mathrm{~min}$, 40 cycles of $\left(94^{\circ} \mathrm{C} 1 \mathrm{~min}, 58^{\circ} \mathrm{C} 50 \mathrm{~s}, 72^{\circ} \mathrm{C}\right.$ for $\left.1 \mathrm{~min}\right)$, $72{ }^{\circ} \mathrm{C} 5 \mathrm{~min}$. Amplified samples were held at $4{ }^{\circ} \mathrm{C}$. Amplification was confirmed by agarose gel electrophoresis of $5 \mu \mathrm{l}$ of each sample.

\section{Single Strand Conformation Polymorphism}

Nine $\mu \mathrm{l}$ of PCR product was mixed with $1 \mu \mathrm{l}$ denaturation solution (0.2 M NaOH, 0.04 M EDTA, pH8). After incubation at $56{ }^{\circ} \mathrm{C}$ for $5 \mathrm{~min}, 2 \mu \mathrm{l}$ formamide loading buffer $(0.05 \%$ bromphenol blue in formamide) was added. Five $\mu$ l of each sample mix was resolved on $12.5 \%$ polyacrylamide at $5{ }^{\circ} \mathrm{C}$. 
Band patterns were visualized using the DNA Silver Staining Kit (GE Healthcare Life Sciences, Pittsburgh, PA) according to manufacturer's directions.

\section{TP53 DNA Sequencing}

Cases that showed TP53 mutation band pattern by SSCP were subject to Sanger sequencing. For sequencing of selected exons, PCR was repeated using a $50 \mu \mathrm{l}$ reaction mix volume. The entire reaction mix was cleaned using S-400 spin columns (GE Healthcare), followed by ethanol precipitation at $-20{ }^{\circ} \mathrm{C}$ for one hour. Pellets were rinsed once in $70 \%$ ethanol and resuspended in $10 \mu \mathrm{l}$ nuclease-free water. Two microliters of template DNA was mixed with $0.5 \mu \mathrm{l}$ forward or reverse primer $(5 \mu \mathrm{M})$; and $4 \mu \mathrm{l}$ Terminator Read y Mix (Applied Biosystems). Cycle sequencing was performed in the ABI 9700 thermal cycler. The program was comprised of $96{ }^{\circ} \mathrm{C}$ one minute followed by 25 cycles of $\left(96{ }^{\circ} \mathrm{C} 10 \mathrm{~s}, 50{ }^{\circ} \mathrm{C} 5 \mathrm{~s}\right.$ and $\left.60^{\circ} \mathrm{C} 4 \mathrm{~min}\right)$. Unincorporated nucleotides were removed using S-200 spin columns (GE Healthcare) followed by ethanol precipitation. The resulting pellets were resuspended in $12 \mu \mathrm{l}$ formamide and resolved by capillary electrophoresis.

\section{Results}

Data was collected from 32 cases, including 19 malignant and 13 benign cases. P53 immunostain was positive in 5 cases, while 27 cases were negative (Table 2). Eight mutation patterns (different from normal control) were detected by SSCP. Five of these were confirmed by sequencing (Figure 1).

Two of three high grade invasive tumors with positive p53 immunostain had a mutation band pattern by SSCP, 2 of which were in exon 6 . TP53exon 6 sequencing confirmed the presence of these mutations (Table 3).

Table 1 Primer sequences for TP53 mutation analysis

\begin{tabular}{lll}
\hline Exon & Forward $\left(5^{\prime}-3^{\prime}\right)$ & Reverse $\left(5^{\prime}-3^{\prime}\right)$ \\
\hline 5 & TTCCTCTTCCTGCAGTACTC & ACCCTGGGCAACCAGCCCTGT \\
6 & ACAGGGCTGGTTGCCCAGGGT & AGTTGCAAACCAGACCTCAG \\
7 & GTGTTGTCTCCTAGGTGGC & GTCAGAGGCAAGCAGAGGCT \\
8 & TATCCTGAGTAGTGGTAATC & AAGTGAATCTGAGGCATAAC \\
9 & GCAGTTATGCCTCAGATTCAC & AAGACTTAGTACCTGAAGGGT
\end{tabular}

Only one malignant case with p53 positive immunostain had a negative SSCP. This case was of a patient with a history of an ovarian mass, with extensive peritoneal involvement and a positive peritoneal fluid cytology for high grade serous carcinoma. However, the patient underwent chemotherapy, and radiation therapy before surgery (total hysterectomy and bilateral salpingo-oophorectomy, omentectomy and lymphadenectomy). Histologically, the only evidence of tumor was few foci of high-grade serous carcinoma present within the lymphatic channels in the peritoneum.

There were 2 histologically benign cases in which p53 immunostain was positive in the fallopian tubes (Figure 2). These lesions represent what is described in the literature as serous tubal intraepithelial

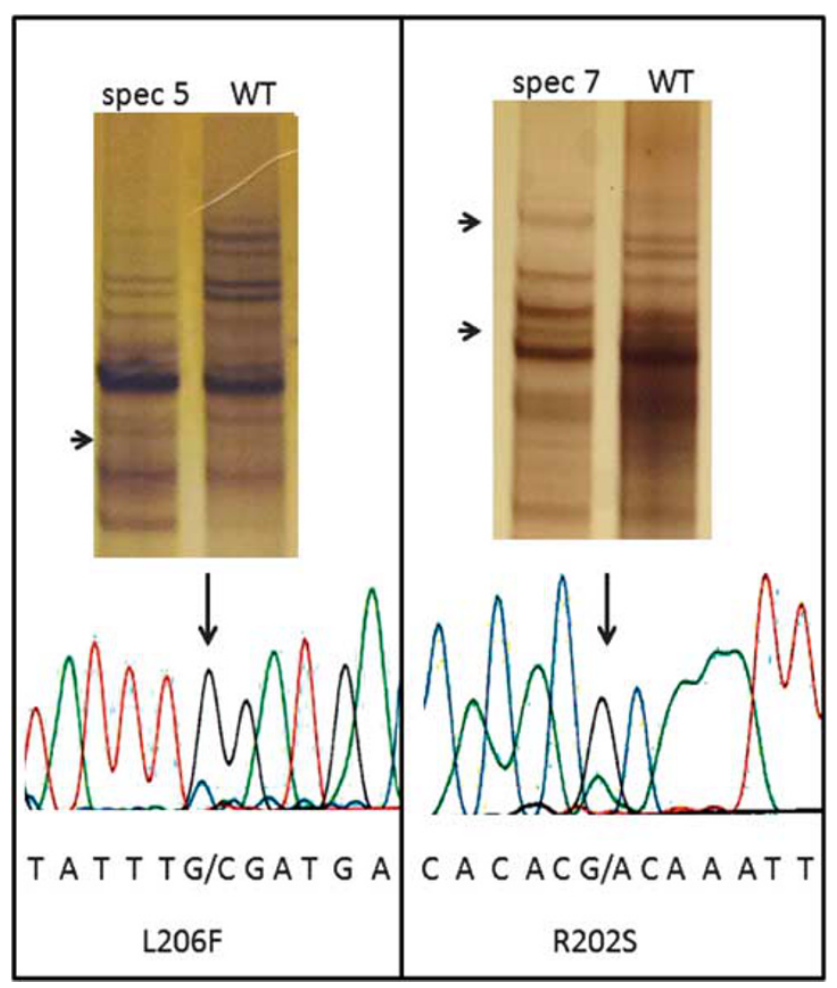

Figure 1 Example data from single-strand conformation polymorphism with sequence confirmation. The band alterations compared to normal patterns (WT) are indicated by arrows. The SSCP mutation detected in specimen 5 was also detected as a leu to phe mutation at amino acid position 206 (left). The SSCP mutation detected in specimen 7 was also detected as an arg to ser mutation at amino acid position 202 (right).

Table 2 Distribution of the positive cases by immunohistochemistry

\begin{tabular}{|c|c|c|c|c|c|c|c|c|}
\hline \multirow{3}{*}{$\begin{array}{l}\text { P53 Immunostain } \\
\text { benign versus malignant } \\
\text { SSCP }\end{array}$} & \multicolumn{4}{|c|}{ Positive $(\mathrm{n}=5)$} & \multicolumn{4}{|c|}{ Negative $(\mathrm{n}=27)$} \\
\hline & \multicolumn{2}{|c|}{$\operatorname{Benign}^{a}(\mathrm{n}=2)$} & \multicolumn{2}{|c|}{ Malignant $(\mathrm{n}=3)$} & \multicolumn{2}{|c|}{ Benign $(\mathrm{n}=11)$} & \multicolumn{2}{|c|}{ Malignant $(\mathrm{n}=16)$} \\
\hline & Negative & Positive & Negative & Positive & Negative & Positive & Negative & Positive \\
\hline Number of cases & 0 & 2 & 1 & 2 & 10 & 1 & 13 & 3 \\
\hline
\end{tabular}

${ }^{\mathrm{a} B o t h}$ cases have serous tubal intaepithelial lesion (STIL) or p53 signature. 
Table 3 Description of the cases that are positive for TP53 mutation band pattern by SSCP method

\begin{tabular}{lllcclcc}
\hline Case & Age & Classification & Grade & Stage & P53 immunostain & Exon mutated by SSCP & Mutation \\
\hline 1 & 53 & EEA & 2 & 1 & Negative & 5 & NI \\
2 & 67 & STIL & NA & 0 & Positive & 6 & NI \\
3 & 49 & EEA & 1 & 1 & Negative & 6 & M215G \\
4 & 63 & MMMT & 3 & 1 & Positive & 6 & P191T \\
5 & 72 & EEA & 1 & 1 & Negative & 6 & N206PF \\
6 & 53 & STIL & NA & 0 & Positive & RI \\
7 & 50 & Normal & NA & NA & Negative & N210H \\
8 & 75 & ESC & 3 & 1 & Positive & & 6 \\
\hline
\end{tabular}

EEA, endometrial endometroid adenocarcinoma; ESC, endometrial serous carcinoma; MMMT, mixed malignant mullerian tumor of the uterine corpus; NA, Not applicable; NI, not identified; STIL, serous tubal intraepithelial lesion.

${ }^{\mathrm{a}}$ This tumor was $2 \mathrm{~mm}$ in size.

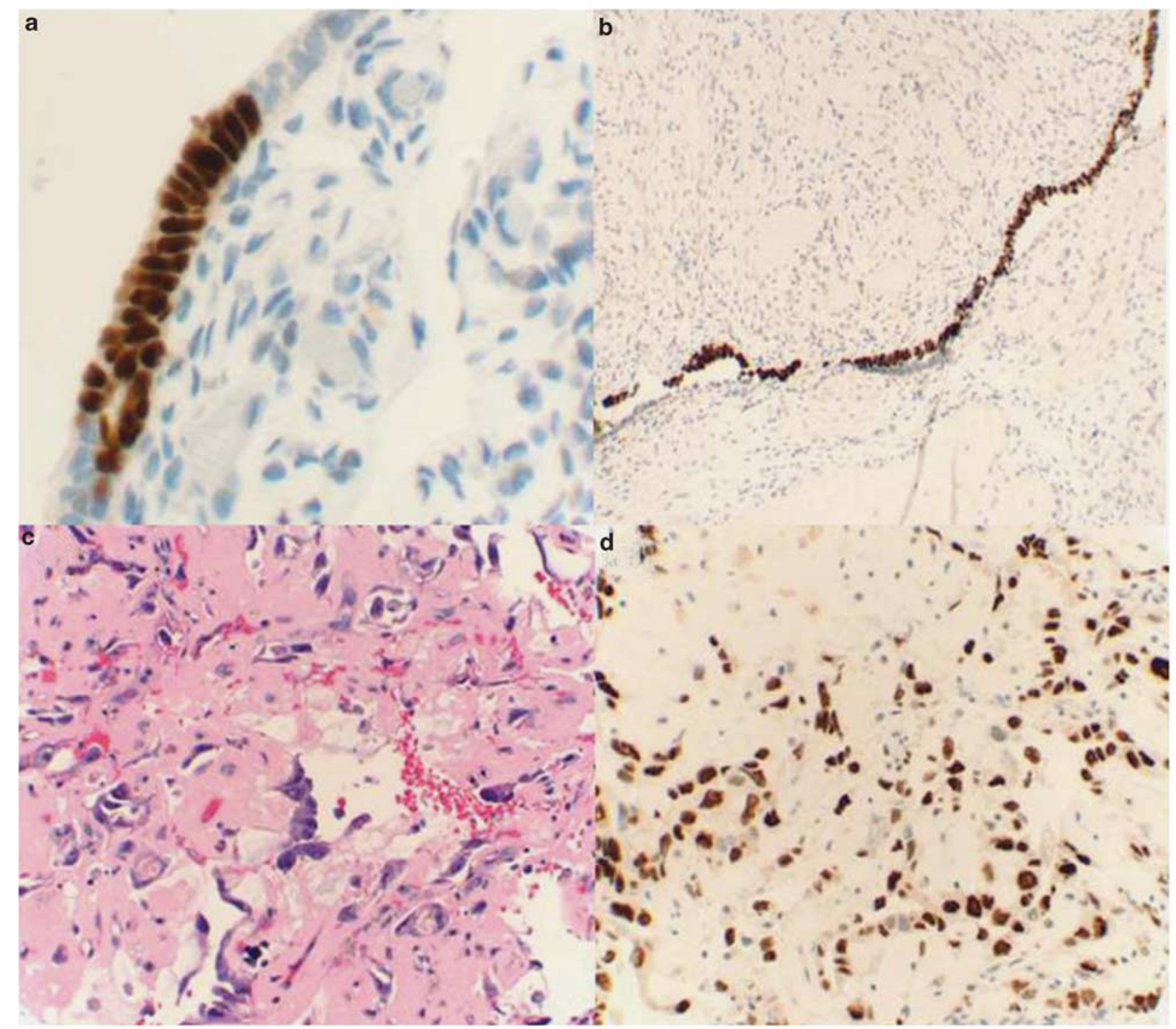

Figure 2 p53 immunostain labeling of serous tubal intraepithelial lesions and small endometrial serous adenocarcinoma. (a) First case of serous tubal intraepithelial lesion (p53 immunostain, 200X). (b) Second case of serous tubal intraepithelial lesion (p53 immunostain, 100X). (c) Small endometrial serous adenocarcinoma (H\&E, 200X). (d) Strong positivity for p53 immunostain in the small endometrial serous adenocarcinoma (p53 immunostain, 200X).

lesions (STILs) or p53 signature, as in the areas were p53 immunostain was positive, the epithelium was not stratified and was histologically normal. These 2 cases showed mutation band patterns in exon 6 by SSCP (Table 3) that were not detected by sequencing. 
From the 15 malignant tumors with negative p53 immunostaining, only 3 cases had a mutation band pattern by SSCP. These cases were FIGO stage I and grade 1-2 endometroid endometrial adenocarcinomas. The patients were 72,53 , and 49 years old.

Among the 11 cases with benign histology and negative p53 immunostain, 1 case had a mutation band pattern in exon 6 by SSCP (Table 3). This case was a 50 year-old patient who underwent total hysterectomy and bilateral salpingo-oophorectomy for a benign condition and had an absolutely benign histology. P53 immunostain was negative in the sections from the fallopian tubes.

Based on this data, the sensitivity, and specificity of SSCP in detecting TP53 mutations in the mucus in high grade malignancies are 80 and $85 \%$ respectively. The sensitivity of SSCP increases to $100 \%$ if we exclude the case of high grade tumor that was confined to the peritoneum, while the specificity remains $85 \%$. However, the sensitivity and specificity of SSCP in detecting only invasive high grade carcinomas are 66 and $79 \%$ respectively.

\section{Discussion}

The results of this study provide sufficient evidence that DNA from high-grade precancerous and cancerous lesions can be detected in the cervical mucus. In addition, SSCP detected potential precancerous lesions in the fallopian tube which suggests that it might detect very early events in fallopian tube and ovarian cancer development. ${ }^{8-10}$ We could not include cases of high-grade carcinomas of the ovary as the cases we received within our inclusion period had involvement of the serosal surface. The only ovarian cancer we could include was a stage 1 clear cell carcinoma that was negative for p53 immunostain, and had no identifiable TP53 mutation pattern by SSCP.

Our main limitation with the results of this study is that it was done in a post operative setting, which could have caused contamination of the cervical mucus despite our cautious handling of the material. However, such contamination is less likely to explain the positive SSCP in patients with precancerous lesions (STIL) in the fallopian tubes or very small malignancies confined to the uterine cavity.

The study included cases of low-grade endometrial endometrioid adenocarcinomas that were p53 negative by immunohistochemistry but showed TP53 mutation in the mucus. This could be explained by the sporadic mutations that occur in cancer cells that might have been detected in the mucus.

One case of a benign uterus showed TP53 mutation by SSCP and DNA sequencing. We think that this patient might have a precancerous lesion that was not detected by routine histology and was not present in the sections stained with p53 antibody.

The second step of this project will be a prospective study. We plan to collect cervical mucus from patients with suspected malignancies and suspected benign diseases in preoperative consultations. We will seek to detect different genetic and environmental markers using molecular genetics methods in conjunction with mass spectrometry. This will help us extend our research to other proteins, and molecules. The theory behind the use of mass spectrometry in analyzing the mucus of cancerous lesion lies in the tumor invasion of the basement membrane into the stroma, which will cause destruction and leakage of connective tissue molecules. In addition, neoplastic epithelium is more friable and apoptotic than the atrophic epithelium of postmenopausal women. The study and analysis of different molecules from epithelium, and stroma in both malignant and benign tumors might allow us to identify profiles that help distinguish normal mucus and mucus contaminated with malignant tumor and its environment. When an adequate database is established, we can initiate the third step of the project which will consist of a large-scale prospective study that will include women over the age of 50 . The mucus sample can be taken in conjuction with routine Pap smears or routine medical checkups. The cases will then be screened using the biomarker(s) and method(s) studied in step 2 that showed the highest sensitivity, and efficiency. The positive cases will be subject to a profiling that will determine the risk of the patient of having a malignancy versus precancerous lesion, its type, and possibly its location.

Although we started our study with minimal resources to explore the possibility to screen for high grade gynecological malignancies using cervical mucus, the recent publication by Kindle I et $a l^{14}$ supports our theory and the findings of our study. Moreover, our results bring additional findings. We demonstrated that precancerous gene mutations could also be detected. The impact of mutational analysis on an eventual screening test using DNA content of cervical mucus still needs to be investigated. Finally, combined efforts of the medical and scientific community could confirm these results and improve the sensitivity of using cervical mucus as a screening test.

\section{Acknowledgements}

Luis Blanco, Matthew Fox, Rohit Singh, Erica Paulsen, Lindsey Franklin and William Barbanera for their help in specimen collection and testing.

\section{Disclosure/conflict of interest}

The authors declare no conflict of interest. 


\section{References}

1 Sankaranarayanan R, Ferlay J. Worldwide burden of gynaecological cancer: the size of the problem. Best Pract Res Clin Obstet Gynaecol 2006;20:207-225.

2 Siegel R, Naishadham D, Jemal A. Cancer statistics. CA Cancer J Clin 2012;62:10-29.

3 McCluggage WG. Morphological subtypes of ovarian carcinoma: a review with emphasis on new developments and pathogenesis. Pathology 2011;43:420-432.

4 Fader AN, Boruta D, Olawaiye AB, et al. Uterine papillary serous carcinoma: Epidemiology, pathogenesis and management. Curr Opin Obstet Gynecol 2010;22:21-29.

5 Kuhn E, Wu RC, Guan B, et al. Identification of molecular pathway aberrations in uterine serous carcinoma by genome-wide analyses. J Natl Cancer Inst 2012;104:1503-1513.

6 Vang R, Shih I, Kurman RJ. Fallopian tube precursors of ovarian low- and high-grade serous neoplasms. Histopathology 2013;62:44-58.

7 Przybycin CG, Kurman RJ, Ronnett BM, et al. Are all pelvic (nonuterine) serous carcinomas of tubal origin? Am J Surg Pathol 2010;34:1407-1416.

8 Kuhn E, Kurman RJ, Vang R, et al. TP53 mutations in serous tubal intraepithelial carcinoma and concurrent pelvic high-grade serous carcinoma-evidence supporting the clonal relationship of the two lesions. J Pathol 2012;226:421-426.

9 Lee Y, Miron A, Drapkin R, et al. A candidate precursor to serous carcinoma that originates in the distal fallopian tube. J Pathol 2007;211:26-35.

10 Kurman RJ, Shih I. Molecular pathogenesis and extraovarian origin of epithelial ovarian cancershifting the paradigm. Hum Pathol 2011;42:918-931.

11 Buys SS, Partridge E, Black A, et al. PLCO Project Team. Effect of screening on ovarian cancer mortality: the prostate, lung, colorectal and ovarian (PLCO) cancer screening randomized controlled trial. JAMA 2011;305:2295-2303.

12 American Cancer Society, Detailed guide: Ovarian cancer-Can ovarian cancer be found early? http:// www.cancer.org/cancer/ovariancancer/detailedguide/ ovarian-cancer-detection.

13 Schorge JO, Hossein Saboorian M, Hynan L, et al. ThinPrep detection of cervical and endometrial adenocarcinoma: A retrospective cohort study. Cancer 2002;96:338-343.

14 Kinde I, Bettegowda C, Wang Y, et al. Evaluation of DNA from the papanicolaou test to detect ovarian and endometrial cancers. Sci Transl Med 2013;5: $167 \mathrm{ra} 4$. 